\title{
MicroRNA-192 inhibits the proliferation, migration and invasion of osteosarcoma cells and promotes apoptosis by targeting matrix metalloproteinase-11
}

\author{
GUOWEI SHANG ${ }^{1}$, YANG MI ${ }^{2}$, YINGWU MEI ${ }^{2}$, GUANGHUI WANG ${ }^{1}$, YADONG WANG ${ }^{2}$, \\ XINJIE LI $^{2}$, YISHENG WANG ${ }^{1}$, YUEBAI $\mathrm{LI}^{2}$ and GUOQIANG ZHAO
}

\author{
${ }^{1}$ Department of Orthopedic Surgery, The First Affiliated Hospital of Zhengzhou University, Zhengzhou, \\ Henan 450052; Departments of ${ }^{2}$ Biochemistry and Molecular Biology and ${ }^{3}$ Microbiology and Immunology, \\ School of Basic Medical Sciences, Zhengzhou University, Zhengzhou, Henan 450001, P.R. China
}

Received April 6, 2017; Accepted January 29, 2018

DOI: $10.3892 / \mathrm{ol} .2018 .8239$

\begin{abstract}
MicroRNAs (miRNAs) are small non-coding RNAs that regulate gene expression during stem cell growth, proliferation and differentiation. miRNAs are also involved in the development and progression of a number of cancer types, including osteosarcoma (OS). miR-192 is significantly downregulated in various tumors, including lung, bladder and rectal cancer. miR-192 expression is associated with the migration and invasion of OS cells. However, the expression of miR-192 and its effects on the development of OS have not been reported. In the present study, the involvement of miR-192 and its molecular mechanisms in the development of OS was investigated. The results indicate that miR-192 expression was significantly downregulated in OS tissues compared with non-tumor tissues $(\mathrm{P}<0.05)$. Next, a miR-192 agomir was transfected into the OS cell line MG-63 to upregulate miR-192. The effects of miR-192 overexpression were then investigated by examining cell proliferation, apoptosis, migration and invasion. Matrix metalloproteinase (MMP)-11 belongs to a family of nine or more highly homologous $\mathrm{Zn}^{2+}$-endopeptidases. It was demonstrated that the mRNA and protein expression of MMP-11 were upregulated in OS tissues compared with non-tumor tissues $(\mathrm{P}<0.05)$. MMP-11 was predicted by TargetScan and miRanda as a miR-192 target, which was confirmed by
\end{abstract}

Correspondence to: Professor Yisheng Wang, Department of Orthopedic Surgery, The First Affiliated Hospital of Zhengzhou University, 1 Jianshe East Road, Zhengzhou, Henan 450052, P.R. China

E-mail: wangyisheng@zzu.edu.cn

Professor Yuebai Li, Department of Biochemistry and Molecular Biology, School of Basic Medical Sciences, Zhengzhou University, 100 Kexue Road, Zhengzhou, Henan 450001, P.R. China

E-mail: liyuebai@zzu.edu.cn

Key words: osteosarcoma, miR-192, matrix metalloproteinase-11, proliferation, apoptosis, invasion, migration western blotting and dual-luciferase assays. Finally, it was demonstrated that the overexpression of miR-192 was able to downregulate MMP-11 expression and reduce proliferation, migration and invasion, and promote apoptosis in OS cells. Together, these data indicate that miR-192 may be a tumor suppressor that inhibits the progression and invasion of OS by targeting MMP-11. Therefore, miR-192 may be useful for the diagnosis and treatment of OS.

\section{Introduction}

Osteosarcoma (OS) is a highly aggressive bone malignancy that is the sixth most common cancer in children and adolescents (1). OS has two peaks of onset: 15-19 years and $\geq 70$ years $(2,3)$. The 5 -year survival rate of primary OS is $\sim 65 \%$. However, OS has a substantially poorer prognosis when associated with fatal lung metastases. The 5-year survival rate of patients with OS metastases is $<20 \%$ despite the use of aggressive chemotherapy and radiotherapy (4), or even when physicians resort to radical surgery (5). As current efforts have failed to obtain impressive achievements in treatment outcomes and quality of life, it is imperative to find new treatment and diagnostic strategies for early OS, discover new therapeutic targets and develop new techniques to improve disease prognosis.

MicroRNAs (miRNAs) have been found to be involved in multiple biological processes, including differentiation, proliferation, developmental timing, apoptosis, transposon silencing and antiviral defense (6). Previous research has confirmed that $>1,000$ miRNAs may be involved in regulating $>50 \%$ of human genes (7). One characteristic of miRNAs is their ability to interact with multiple targets. Each miRNA can regulate hundreds of protein-encoding genes and vice versa, as each structural gene can be a target for multiple miRNAs (8). The incorporation of miRNAs in the study of tumor progression and pathogenesis has reached new levels in recent years for various malignancies, including breast, colon, bladder, pancreatic and lung cancer (9-12). However, the field of miRNAs in OS is still in its infancy. To date, miR-RNAs, including miR-124, miR-30a, miR-144, miR-202 and miR-300 have 
been demonstrated to be closely associated with the initiation and progression of OS (13-17). A recent study reported that miR-192 was abnormally expressed in a variety of carcinomas, including OS (18). However, the exact role of miR-192 in OS remains unknown.

Matrix metalloproteinase (MMP)-11 is a member of the MMP family that have important roles in tumor invasion and metastasis, participating in all aspects of the formation and progression of digestive tract, breast and ovarian tumors (19-21). MMP-11 overexpression in primary tumors is associated with poor prognoses (22). Previous studies have shown that MMP-11 is associated with the pathogenesis of various types of cancer, and therefore it is a potential therapeutic target and/or tumor biomarker.

In the present study, the potential association between miR-192 expression, MMP-11 expression and the grade of malignancy in OS cells were investigated. The data indicated that the upregulation of miR-192 inhibited the proliferation, migration and invasion of the OS cell line MG63. Furthermore, it was demonstrated that MMP-11 was downregulated in MG63 cells. Bioinformatic analysis, reverse transcription-quantitative polymerase chain reaction, western blotting and luciferase reporter assays were performed to elucidate the underlying mechanism through which miR-192 targets MMP-11 in OS cells.

\section{Materials and methods}

Collection of patient samples. OS specimens $(n=22)$ were obtained from eligible patients treated at the First Affiliated Hospital of Zhengzhou University (Zhengzhou, China) between June 2014 and May 2016. Tumor and matched adjacent non-cancerous tissue samples were collected and characterized based on histopathological examination. All tissues are snap frozen in liquid nitrogen immediately following surgery and stored at $-80^{\circ} \mathrm{C}$ until use. Prior to the use of these clinical materials for research purposes, written informed consent was obtained from all patients, and the present study was approved by the Institute Research Medical Ethics Committee of Zhengzhou University.

RNA extraction and reverse transcription-quantitative polymerase chain reaction ( $R T-q P C R)$. Total RNA from tissue samples and cultured cells was isolated using TRIzol reagent (Invitrogen; Thermo Fisher Scientific, Inc., Waltham, MA, USA) according to the manufacturer's protocol. cDNA was synthesized using the RevertAid First Strand cDNA kit (Thermo Fisher Scientific, Inc.). RT-qPCR was performed using SYBR-Green Premix Ex Taq (Takara Bio, Inc.) to quantify miR-192 and MMP-11 expression by ABI 7500 fast apparatus, thermocycling conditions: $95^{\circ} \mathrm{C}, 5 \mathrm{~min}$; $95^{\circ} \mathrm{C}, 5 \mathrm{~min} ; 40$ cycles, $95^{\circ} \mathrm{C}, 15 \mathrm{sec} ; 60^{\circ} \mathrm{C}, 30 \mathrm{sec}$. U6 small nuclear RNA and GADPH were used as endogenous controls. The premier sequences was has-miR-192: Forward 5'-GCGGCGGCTGACCTATGAATTG-3'; and reverse: 5'-ATCCAGTGCAGGGTCCGAGG-3'; the primer sequences of U6 were, forward: 5'-TCCGATCGTGAAGCGTTC-3'; and reverse: 5'-GTGCAGGGTCCGAGGT-3'; the primer sequences of GADPH forward: 5'-GAAGGTGAAGGTCGG AGTC-3' and reverse: 5'-ATCCAGTGCAGGGTCCGAGG-3'; the premier sequences of MMP-11 were forward: 5'-CCTAAA GGTATGGAGCGATGT-3' and reverse: 5'-CGATAGTCC AGGTCTCATCAT-3'. The RT-qPCR results were compared using the $2^{-\triangle \Delta C q}$ method (23). The experiments were repeated in three times.

Cell culture. The human OS cell line MG63 was obtained from the Chinese Academy of Medical Sciences (Beijing, China). MG63 cells were maintained in RPMI-1640 medium that was supplemented with $10 \%$ fetal bovine serum (FBS; Sigma-Aldrich; Merck KGaA, Darmstadt, Germany), 100 U/ml penicillin and $100 \mu \mathrm{g} / \mathrm{ml}$ streptomycin at $37^{\circ} \mathrm{C}$ in a $5 \% \mathrm{CO}_{2}$ humidified atmosphere. Human osteoblast hFOB1.19 cells were cultured in Dulbecco's modified Eagle's medium supplemented with $10 \%$ FBS (Gibco; Thermo Fisher Scientific, Inc.), $100 \mathrm{U} / \mathrm{ml}$ penicillin and $100 \mu \mathrm{g} / \mathrm{ml}$ streptomycin at $37^{\circ} \mathrm{C}$ in a $5 \% \mathrm{CO}_{2}$ humidified atmosphere.

Transfection of miRNA. MG-63 cells were seeded into each well of a 6 -well plate $\left(2 \times 10^{5}\right.$ cells/well) and then transfected with either miR-192 agomir or negative control (NC), or miR-192 antagomir or NC (Shanghai GenePharma Co., Ltd., Shanghai, China) (50 nM) using Lipofectamine ${ }^{\circledR} 2000$ (Invitrogen; Thermo Fisher Scientific, Inc.,) when the cell confluence was $\sim 90 \%$. The cells were harvested for further experiments $24-48 \mathrm{~h}$ following transfection.

Cell proliferation and apoptosis assays. Cell proliferation was analyzed using Cell Counting Kit-8 (CCK-8; Dojindo Molecular Technologies, Inc., Kumamoto, Japan). The transfected MG63 cells $\left(5 \times 10^{3}\right.$ cells/well) were seeded into 96-well plates in a final volume of $100 \mu \mathrm{l}$ and cultured for $24,48,72$, 96 and $120 \mathrm{~h}$. The CCK-8 solution $(10 \mu \mathrm{l})$ was added into each well, incubated for $4 \mathrm{~h}$, and the optical density at $450 \mathrm{~nm}$ was measured to calculate the number of viable cells. A total of five parallel wells were analyzed for each group. The apoptosis assay was conducted using caspase- 3 analysis. The cells from each group were harvested 36-h post-transfection by trypsinization. The cells were then seeded into 96 -well plates at a total density of $2 \times 10^{6}$ cells/well in a mixed Ac-DEVD-PNA buffer and incubated at $37^{\circ} \mathrm{C}$ overnight. On the following day, absorbance values were measured at $415 \mathrm{~nm}$.

Transwell assays. The cells from each group were collected 36 -h post-transfection and resuspended in medium at $2 \times 10^{5}$ cells $/ \mathrm{ml}$. The upper chamber of a 24-well Transwell Permeable Support device (Corning, Corning, NY, USA) with $8-\mu \mathrm{m}$ pores was loaded with $200 \mu \mathrm{l}$ cell suspension, and the lower chamber was supplied with $500 \mu$ l complete medium (RPMI-1640) containing 10\% FBS. The loaded device was incubated at $37^{\circ} \mathrm{C}$ in a humidified incubator with $5 \% \mathrm{CO}_{2}$ for $36 \mathrm{~h}$. After incubation, the medium was removed from the upper chamber and the cells in this chamber were scraped off with a cotton swab. The cells that had migrated to the other side of the membrane were fixed with methanol. The cells were then stained with hematoxylin, mounted and dried at $80^{\circ} \mathrm{C}$ for $30 \mathrm{~min}$. The number of invaded cells in three randomly selected fields was counted using an inverted microscope at a magnification of $\mathrm{x} 400$. The experiments were performed in triplicate. 
Wound-healing assays. The cells from each group were collected 24-h post-transfection, seeded in 6-well plates $\left(2 \times 10^{3}\right.$ cells/well) and allowed to adhere for a further $24 \mathrm{~h}$. An artificial wound was made using a $10-\mu 1$ pipette tip across the cell monolayer. The cells were then rinsed with phosphate-buffered saline (PBS) three times to clear cell debris and cells remaining in suspension, and then cultured in serum-free RPMI-1640 medium. Micrographs (taken using an inverted microscope) of the same position relative to the wound were taken at 0 and $36 \mathrm{~h}$. Each assay was performed in triplicate.

Target prediction. Bioinformatic analysis was conducted using TargetScan (http://www.targetscan.org/vert_71/) and miRanda (http://34.236.212.39/microrna/home.do) to predict the target gene of miR-192 and to verify MMP-11 3'UTR containing the seed region of miR-192.

Luciferase reporter assays. Regions of the human MMP-11 gene containing putative miR-192 binding sites were amplified from human genomic DNA by RT-PCR, performed as previously mentioned. These fragments were cloned into the pmirGLO vector (Promega Corporation, Madison, WI, USA) downstream of the luciferase gene to generate the recombinant vectors pGL3-MMP-11-wt and pGL3-MMP-11-mut. For luciferase reporter assays, a Dual-Luciferase Reporter Assay system (Promega Corporation) was used. MG63 cells were cultured in 96-well plates and co-transfected using 0.8 pmol Pmir-Glo with $50 \mathrm{nM}$ miRNA and wild-type or mutant reporter vectors using Lipofectamine ${ }^{\circledR} 2000$. Luciferase values were determined using the Dual-Luciferase Reporter Assay system (Promega Corporation) 24 h post-transfection.

Western blotting. RIPA buffer was prepared for protein isolation, and the cell lysis solution was centrifuged at $13,000 \times \mathrm{g}$ for $5 \mathrm{~min}$ at $4^{\circ} \mathrm{C}$, and then the supernatants were collected. Protein concentration was determined using the BCA assay. Proteins were loaded for SDS-PAGE on $10 \%$ polyacrylamide gels followed by transfer to polyvinylidene difluoride membranes. The membrane was blocked for $0.5 \mathrm{~h}$ at room temperature in $5 \%$ skimmed milk, followed by incubation with primary antibodies (1:300; Ruiqi Biotechnology, Shanghai, China) at $4^{\circ} \mathrm{C}$ overnight. After washing three times with TBST (5 min each), the membranes were incubated with a 1:2,500 dilution of goat anti-mouse IgG (Santa Cruz Biotechnology, Inc., Dallas, TX, USA) secondary antibody for $1 \mathrm{~h}$ at room temperature and washed again. Immunoblotting was performed using a chemiluminescence detection kit (Amersham Pharmacia Biotech Inc., Piscataway, NJ, USA). The signals from target proteins were normalized to those of GAPDH. The software used for densitometric analysis was Image J (version 1.8.0_112; National Institutes of Health, Bethesda, MD, USA).

Statistical analysis. All analyses were performed using SPSS (version 17.0; SPSS, Inc., Chicago, IL, USA). Data are expressed as the mean \pm standard deviation. The differences were evaluated using a paired Student's t-test or one-way analysis of variance as appropriate. A value of $\mathrm{P}<0.05$ was used to indicate a statistically significant difference.

\section{Results}

Inverse correlation between miR-192 and MMP-11 expression in human OS tissues and cell lines. The levels of miR-192 and MMP-11 expression were detected by RT-qPCR in human OS and matched adjacent non-tumor tissues as well as a normal human osteoblastic cell line (hFOB1.19) and an OS cell line (MG63) in order to investigate the role of miR-192 in the development of human OS. These results showed that miR-192 expression was significantly downregulated in OS tissues compared with matched adjacent non-tumor tissues $(\mathrm{P}<0.05$; Fig. 1A). By contrast, the levels of MMP-11 mRNA were higher in OS tissues compared with matched adjacent non-tumor tissue $(\mathrm{P}<0.05$; Fig. 1A). Similarly, the level of miR-192 expression in MG63 cells was lower compared with hFOB1.19 cells. Conversely, MMP-11 mRNA was upregulated in MG63 cells compared with hFOB1.19 cells $(\mathrm{P}<0.05$; Fig. 1B). Furthermore, western blotting showed that MMP-11 protein levels were also markedly upregulated in OS tissues compared with matched adjacent non-tumor tissues, and in MG63 cells compared with hFOB1.19 cells (Fig. 1C). These data revealed that there is an inverse correlation between the expression of miR192 and MMP-11 in OS tissues and matched adjacent non-tumor tissues (Fig. 1D).

Upregulation of miR-192 inhibits proliferation and induces apoptosis in MG63 cells. To investigate the functional roles of miR-192, miR-192 agomir or NC constructs were transfected into MG63 cells and miR-192 expression was detected after 36 h. Subsequently, CCK- 8 and caspase-3 assays were conducted to evaluate the changes in proliferation and apoptosis, respectively. RT-qPCR analysis revealed that miR-192 expression was increased by transfection of miR-192 agomir compared with the control group $(\mathrm{P}<0.01$; Fig. $2 \mathrm{~A})$. The CCK-8 assay showed that transfection of miR-192 agomir was able to significantly suppress the proliferation of MG63 cells at five consecutive time points compared with the NC and control groups (Fig. 2B). Moreover, the caspase-3 assay indicated that the upregulation of miR-192 in MG-63 cells was able to promote apoptosis compared with the $\mathrm{NC}$ and control groups $(\mathrm{P}<0.05$; Fig. 2C).

Downregulation of miR-192 promotes proliferation and suppresses apoptosis in MG63 cells. Next, the NC or miR-192 antagomir was transfected into MG63 cells. Following incubation for $36 \mathrm{~h}$, miR-192 expression, cell proliferation and apoptosis were detected. miR192 expression was sharply decreased by miR-192 antagomir transfection compared with the control ( $\mathrm{P}<0.01$; Fig. 3A). The miR-192 antagomir was also able to markedly promote proliferation (Fig. 3B) and significantly block apoptosis in MG63 cells $(\mathrm{P}<0.05$; Fig. 3C). These data indicated that miR-192 had a strong effect on the proliferation and apoptosis of OS cells.

Effects of miR-192 on the migration and invasion of MG63 cells. Next, the effects of miR-192 on the migration and invasion of MG63 cells were investigated. In wound healing assays, the upregulation of miR-192 was able to suppress the 
A

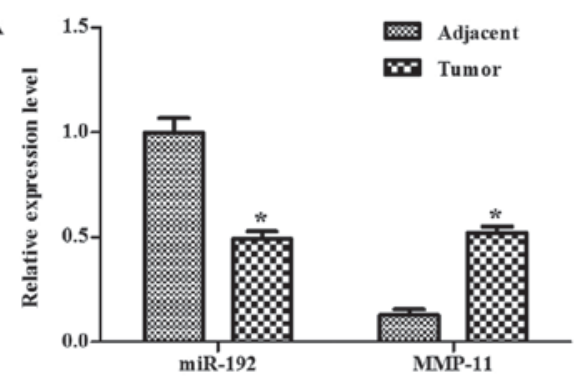

C

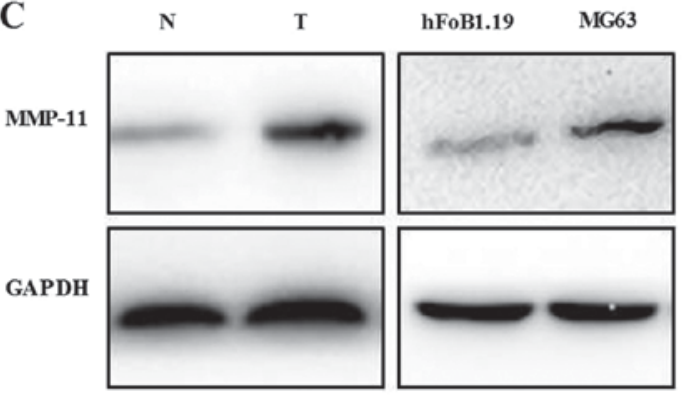

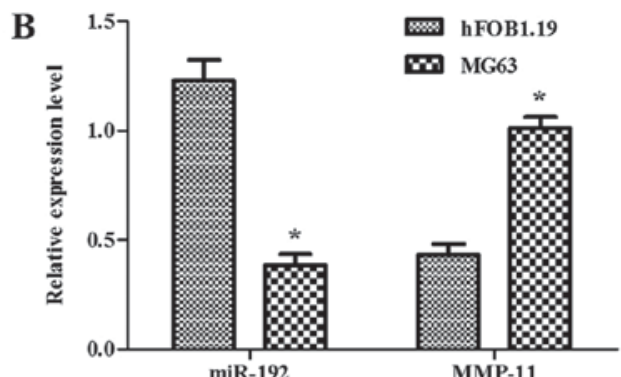

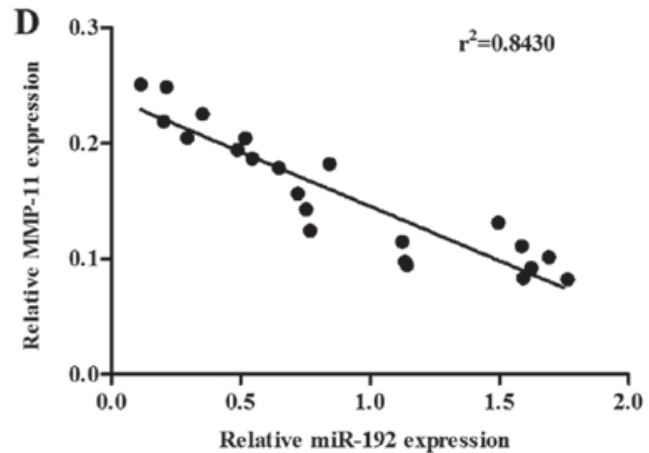

Figure 1. miR-192 and MMP-11 expression levels were detected in OS tissues and cell lines. (A) miR-192 expression was downregulated in OS tissues compared with matched normal adjacent tissues. MMP-11 expression was higher in OS tissues compared with normal adjacent tissues. ${ }^{*} \mathrm{P}<0.05$ vs. adjacent normal tissues (B) miR-192 expression was suppressed in MG63 cells compared with hFOB1.19 cells; MMP-11 expression was increased in MG63 cells compared with hFOB1.19 cells. "P<0.05 vs. hFOB1.19 cells. (C) Western blotting was performed to detect the levels of MMP-11 protein in OS tissues and cell lines with GADPH as a loading control. N, adjacent normal tissues; T, tumor tissues. (D) Analysis of the correlation between miR-192 and MMP-11 expression levels in OS tissues. MMP-11, matrix metalloproteinase-11, miRNA, microRNA; OS, osteosarcoma.

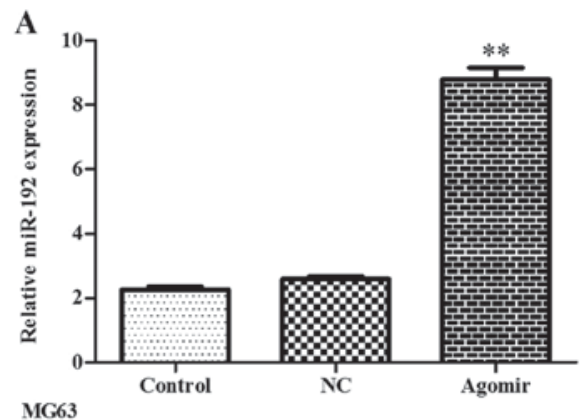

MG63

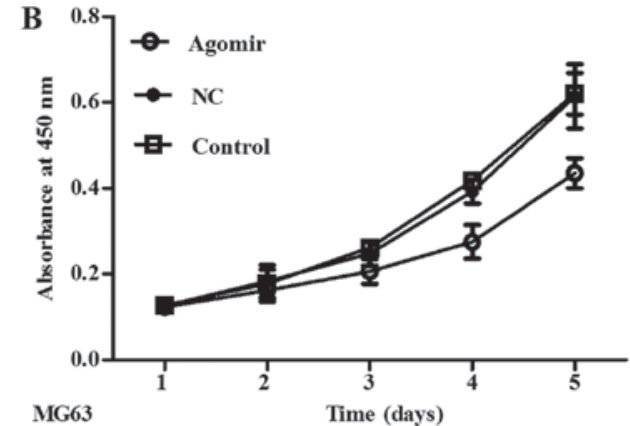

MG63

C

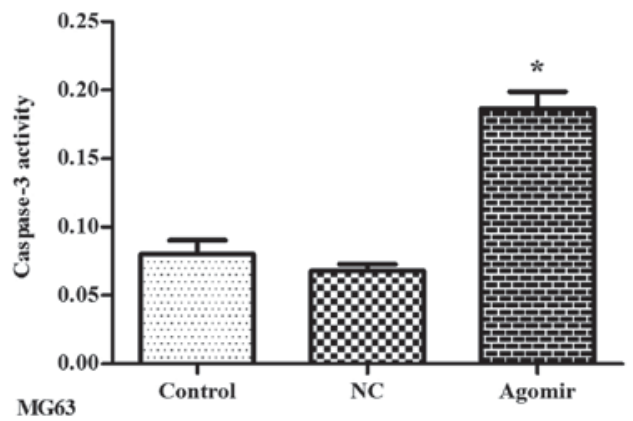

Figure 2. miR-192 overexpression inhibits proliferation and increases apoptosis in MG63 cells. (A) The miR-192 agomir was transfected into MG63 cells. (B) Cell proliferation was analyzed using Cell-Counting Kit 8 assay following transfection in MG63 cells. (C) Apoptosis was detected with caspase-3 assay. ${ }^{*} \mathrm{P}<0.05,{ }^{* *} \mathrm{P}<0.01$ vs. control. $\mathrm{NC}$, negative control.

migration of MG63 cells to a significant extent, as observed by the degree of wound closure after $36 \mathrm{~h}$ under a microscope (Fig. 4A). Similarly, Transwell assays indicated that the overexpression of miR-192 led to a significantly decreased invasive ability of MG63 cells compared with the NC and control groups (Fig. 4B). Then, miR-192 expression was decreased by transfection of miR-192 antagomir, and wound healing and Transwell assays were performed. After $36 \mathrm{~h}$, the 

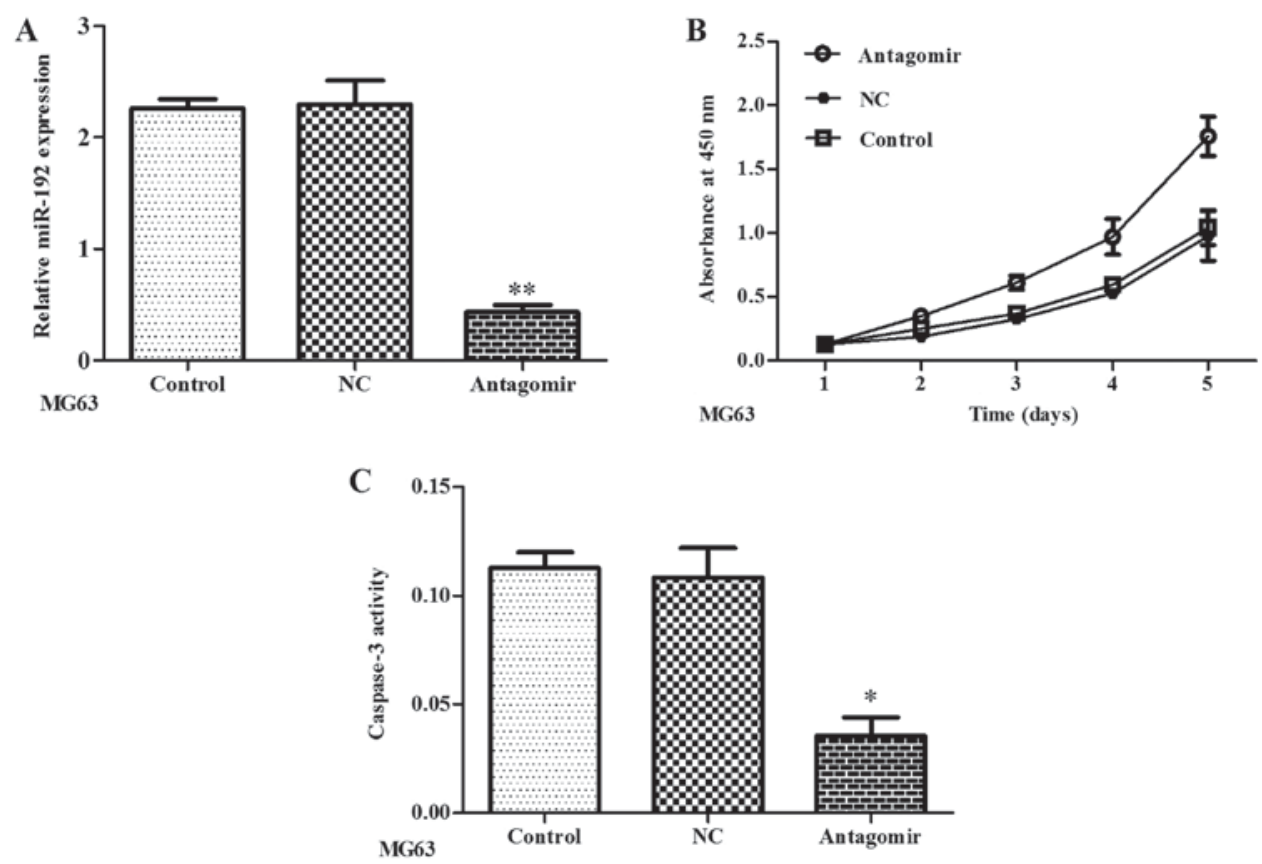

Figure 3. miR-192 downregulation increases proliferation and suppresses apoptosis in MG63 cells. (A) The miR-192 antagomir was transfected into MG63 cells. (B) Cell proliferation was measured via Cell-Counting Kit 8 assay following transfection in MG63 cells. (C) Apoptosis was detected by caspase-3 assay following transfection in MG63 cells. ${ }^{*} \mathrm{P}<0.05,{ }^{* *} \mathrm{P}<0.01$ vs. control. NC, negative control; miR, mircoRNA.

A

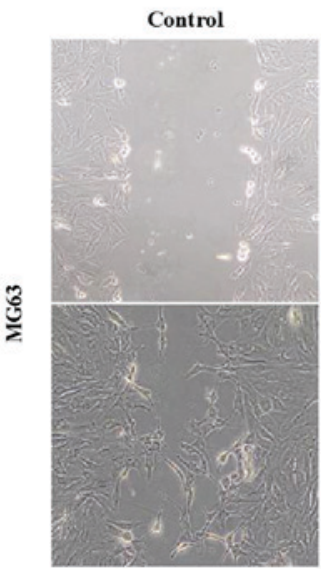

B

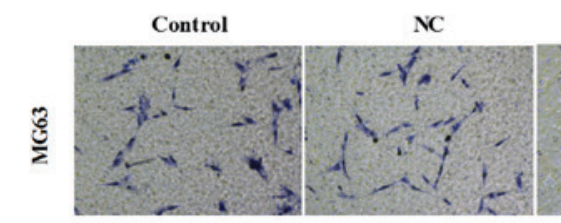

$\mathrm{NC}$

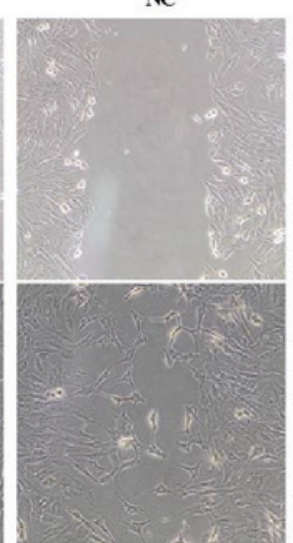

miR-192 agomir

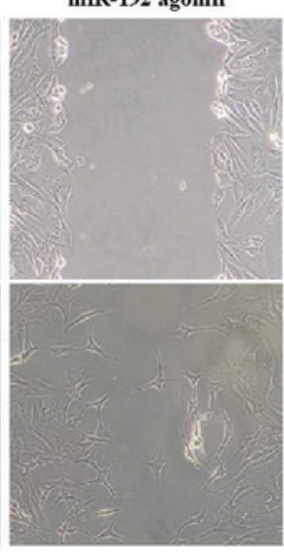

miR-192 agomir
miR-192 antagomir

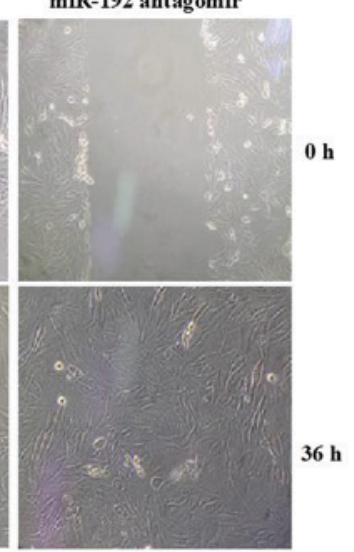

miR-192 antagomir
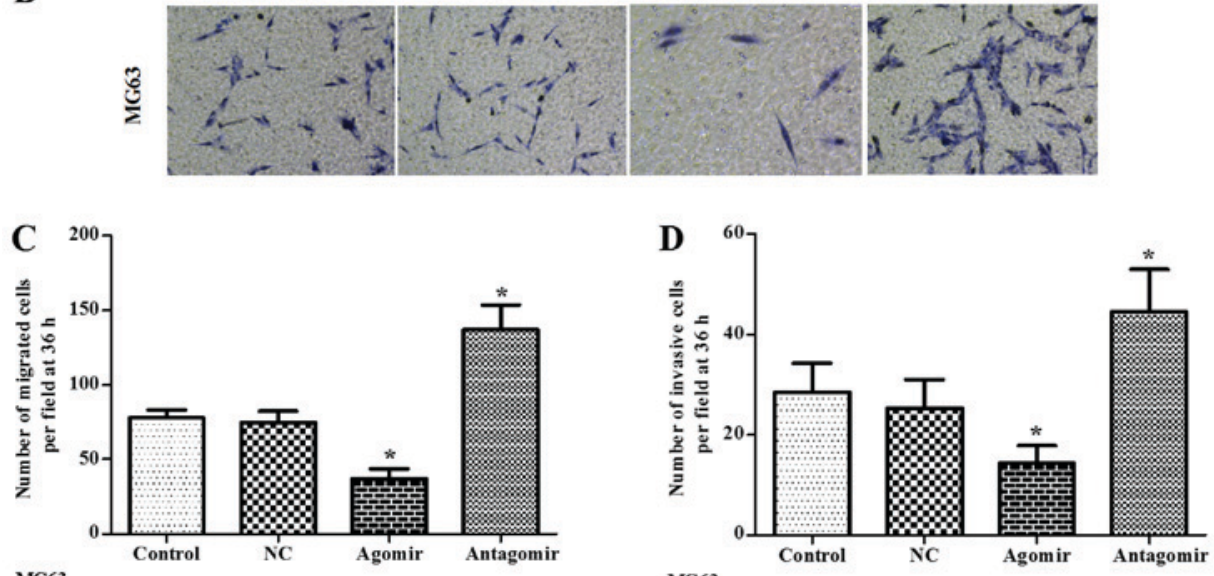

MG63

Figure 4. miR-192 agomir and antagomir modulate the migration and invasion of MG63 cells. The miR-192 agomir or NC and miR-192 antagomir or NC were transfected into MG63 cells. (A) Effect of miR-192 on the migration of MG63 cells as assessed by wound-healing assay. Representative images for each group were taken using a light microscope. Magnification, x200. (B) Effect of miR-192 on the invasion of MG63 cells as assessed by Transwell assay. Representative images for each group were evaluated by light microscopy. Magnification, x400. (C) The migrated cells in each field were counted and analyzed. (D) The invaded cells in each field were counted and analyzed. ${ }^{*} \mathrm{P}<0.05$ vs. control. miRNA, microRNA; NC, negative control. 

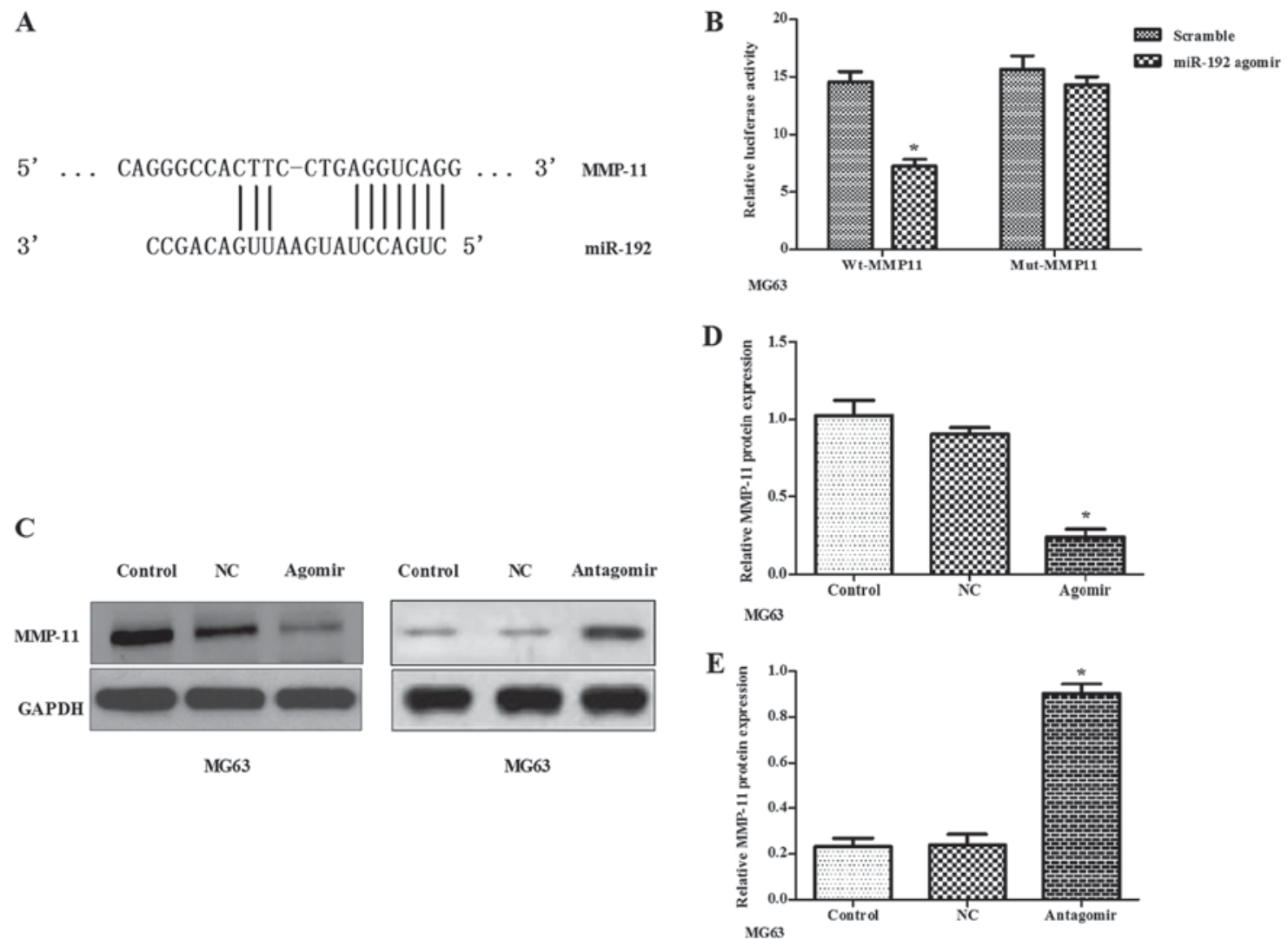

Figure 5. miR-192 suppresses MMP-11 expression by directly targeting the 3'-UTR of MMP-11 mRNA. (A) Putative miR-192 binding sequences in the MMP-11 3'-UTR. (B) Luciferase activity in transfected cells $48 \mathrm{~h}$ after transfection. WT-MMP-11, pGL3-MMP-11-WT; MUT-MMP-11, pGL3-MMP-11-MUT. ${ }^{*} \mathrm{P}<0.05$ vs. the scramble group. (C) Western blot analysis of MMP-11 protein levels in transfected MG63 cells with GADPH as a loading control. Control. non-transfected cells, NC, cells that were transfected with a scrambled miR-192 sequence, agomir and antagomir, cells that are transfected with miR-192 agomir and miR-192 antagomir, respectively. (D) The transfection of miR-192 agomir resulted in a decrease in MMP-11 protein levels. "P<0.05 vs. control. (E) The miR-192 antagomir upregulated the levels of MMP-11 protein. "P<0.05 vs. control. MMP-11, matrix metalloproteinase-11; miRNA, microRNA; NC, negative control; WT, wild-type; MUT, mutated.

downregulation of miR-192 was able to promote the migration and invasion of MG63 cells, which was on the contrary to the miR-192 overexpression results (Fig. 4A and B). Moreover, statistical analyses demonstrated that the overexpression of miR-192 was able to suppress the migration and invasion of MG63 cells. By contrast, the inhibition of miR-192 enhanced migration and invasion ( $\mathrm{P}<0.05$; Fig. $4 \mathrm{C}$ and $\mathrm{D})$.

MMP-11 is a potential target of miR-192. To gain further insight into the molecular mechanism through which miR-192 modulates the behavior of OS cells, target genes of miR-192 were searched using two commonly used target prediction programs, TargetScan (http://www.targetscan.org/vert_71/) and miRanda (http://34.236.212.39/microrna/home.do). The results showed that MMP-11 was a potential miR-192 target gene (Fig. 5A). To confirm that MMP-11 is a direct miR-192 target, dual-luciferase reporter plasmids containing the 3'-UTR of MMP-11 with either a wild-type or mutant putative miR-192 binding site were constructed. Following the overexpression of miR-192 in MG63 cells, the relative luciferase activity of the construct containing the wild-type 3'-UTR of MMP-11 was significantly downregulated compared with the mutant MMP-11 3'-UTR (Fig. 5B). In subsequent western blot assays, MMP-11 protein levels were decreased in MG63 cells when miR-192 expression was upregulated via transfection of agomir
(Fig. 5C-E). These results suggested that miR-192 suppressed MMP-11 expression by directly targeting its 3'-UTR.

\section{Discussion}

OS is one of the most prevalent human sarcomas (24), but even with the development of new therapeutic technologies, the overall survival of OS patients remains unsatisfactory (3). The highly complex molecular mechanisms underlying the initiation and progression of OS remain unknown. Recent studies have shown that miRNAs, including miRNA-34a, miRNA-145 and miRNA-206, are involved in the development of OS (25).

miRNAs are small non-coding RNA molecules ranging from 17 to $22 \mathrm{bp}$ (26) that function in mRNA silencing and the post-transcriptional regulation of gene expression (27). Recent studies have demonstrated that miRNAs are involved in diverse biological processes, including tumorigenesis $(28,29)$. Each miRNA can control hundreds of gene targets, indicating that miRNAs may function as tumor suppressors or oncogenes via cellular pathways (8). A recent study reported that some miRNAs are dysregulated in cancer. The aberrant expression of these miRNAs may promote tumor proliferation or repress tumor development (30). Therefore, it is necessary to elucidate the mechanism of miRNA-mediated OS development. The present study focused on the roles of miRNA-192 in OS. The 
dysregulation of miRNA-192 has been detected in man types of cancer. Specifically, miR-192 overexpression has been shown to induce apoptosis in bladder cancer cells, suggesting that it may be a tumor suppressor in bladder cancer by regulating cell cycle progression (31). Upregulated miR-192 has also been demonstrated to inhibit the progression and metastases of colon cancer (32). Moreover, elevated miR-192 expression has been reported to cause cell growth arrest in breast cancer cells (33). However, it has been reported that miR-192 is overexpressed in nasopharyngeal carcinoma (34), esophageal cancer (35) and pancreatic ductal adenocarcinoma (36). To date, it is unclear whether the downregulation or upregulation of miR-192 is associated with the development of OS.

In the present study, it was detected that miR-192 was downregulated in clinical human OS specimens and the OS cell line MG63 compared with adjacent tissues or normal cells. Furthermore, the overexpression of miR-192 was able to decrease proliferation and promote apoptosis in MG63 cells. It was also determined that the upregulation of miR-192 was able to suppress the migration and invasion of MG63 cells. Therefore, miR-192 could be an inhibitor of OS metastasis. Taken together, our data suggest that miR-192 is a tumor suppressor in OS. Using a combination of two publicly available bioinformatic algorithms, putative miR-192 binding sites in the 3'-UTR of MMP-11 were detected. Moreover, it was detected that MMP-11 expression was higher in OS tissues compared with matched adjacent normal tissues, and MMP-11 expression was also increased in MG63 cells compared with the normal human osteoblastic cell line hFOB1.19.

MMP-11 is a member of the MMP family, one of the major classes of proteolytic enzymes involved in embryonic development, implantation and organ formation (37). MMP-11 also has a potential correlation with poor prognosis in OS (22). Notably, an inverse correlation between miR-192 expression and MMP-11 in MG63 cells was detected. To further investigate whether MMP-11 is a downstream miR-192 target, dual-luciferase assays were conducted. The overexpression of miR-192 led to a significant decrease in luciferase reporter activity in cells that express wild-type but not mutant MMP-11 3'-UTR constructs. Therefore, MMP-11 may be a direct target of miR-192. These data indicate that the overexpression of miR-192 inhibits the progression and invasion of OS by downregulating MMP-11 expression via directly targeting its 3'UTR. In the future, this could be the basis for anti-metastatic therapy for OS. In light of the principle of miRNA-target recognition, a specific miRNA could have $>100$ targets (38). Therefore, the complex molecular network of miR-192 remains an enormous subject for future investigation.

The present study is the first to demonstrate the crosstalk between miR-192 and MMP-11 in OS. Our findings suggest that miR-192 is downregulated in OS, which plays important roles in meditating the proliferation, apoptosis, migration and invasion of OS cells by allowing the expression of MMP-11. Accordingly, we propose that both miR-192 and MMP-11 could be promising candidate targets for treating OS.

\section{Acknowledgements}

Not applicable.

\section{Funding}

No funding was received.

\section{Availability of data and materials}

The datasets used and analyzed during the current study are available from the corresponding author on reasonable request.

\section{Authors' contributions}

GWS, YSW, GQZ and YBL conceived and designed the experiments; GWS, YM, YWM, GHW and YDW performed the experiments; GWS, YM, YWM, XJL, GHW and YDW analyzed the data; YSW and YBL contributed reagents, materials and apparatus; GWS wrote the paper; YSW and YBL revised this paper; GQZ reviewed this paper.

\section{Ethics approval and consent to participate}

The Institute Research Medical Ethics Committee of Zhengzhou University approved the present study and written informed consent was obtained from each patient.

\section{Consent for publication}

Informed consent was obtained from all individual participants included in the study.

\section{Competing interests}

The authors declare that they have no competing interests.

\section{References}

1. Longhi A, Errani C, De Paolis M Mercuri M and Bacci G: Primary bone osteosarcoma in the pediatric age: State of the art. Cancer Treat Rev 32: 423-436, 2006.

2. Ta HT, Dass CR, Choong PF and Dunstan DE: Osteosarcoma treatment: State of the art. Cancer Metastasis Rev 28: 247-263, 2009.

3. Mirabello L, Troisi RJ and Savage SA: Osteosarcoma incidence and survival rates from 1973 to 2004: Data from the surveillance, epidemiology, and end results program. Cancer 115: 1531-4153, 2009.

4. Hughes DP: Strategies for the targeted delivery of therapeutics for osteosarcoma. Exper Opin Drug Deliv 6: 1311-1321, 2009.

5. Ferguson WS and Goorin AM: Current treatment of osteosarcoma. Cancer Invest 19: 292-315. 2001.

6. Dickey LL, Worne CL, Glover JL, Lane TE and O'Connell RM: MicroRNA-155 enhances T cell trafficking and antiviral effector function in a model of coronavirus-induced neurologic disease. J Nueuroinflammation 13: 240, 2016.

7. Hammond SM: An overview of microRNAs. Adv Drug Deliv Rev 87: 3-14, 2015.

8. Knirsh R, Ben Dror I, Modai S, Shomron N and Vardimon L: MicroRNA $10 \mathrm{~b}$ promotes abnormal expression of the proto-oncogene c-Jun in metastatic breast cancer cells. Oncotarget 7: 59932-59944, 2016.

9. Vychytilova-Faltejskova P, Radova L, Sachlova M, Kosarova Z, Slaba K, Fabian P, Grolich T, Prochazka V, Kala Z, Svoboda M, et al: Serum-based MicroRNA signatures in early diagnosis and prognosis prediction of colon cancer. Carcinogenesis 37: 941-950, 2016.

10. Long Y, Wu Z, Yang X, Chen L, Han Z, Zhang Y, Liu J, Liu W and Liu X: MicroRNA-101 inhibits the proliferation and invasion of bladder cancer cells via targeting c-FOS. Mol Med Rep 14: 2651-2656, 2016 
11. Dong Q, Li C, Che X, Qu J, Fan Y, Li X, Li Y, Wang Q, Liu Y, Yang $\mathrm{X}$ and Qu X: MicroRNA-891b is an independent prognostic factor of pancreatic cancer by targeting Cbl-b to suppress the growth of pancreatic cancer cells. Oncotarget 7: 82338-82353, 2016.

12. Mizuno K, Mataki H, Seki N, Kumamoto T, Kamikawaji K and Inoue H: MicroRNAs in non-small cell lung cancer and idiopathic pulmonary fibrosis. J Hum Genet 62: 57-65, 2017.

13. Karbasy SH, Taheriazam A, Mirghasemi A, Sedaghati F, Shakeri M, Yahaghi E and Bahador R: Retracted Article: Upregulation of miR-300 and downregulation of miR-125b act as potential predictor biomarkers in progression, metastasis, and poor prognosis of osteosarcoma. Tumour Biol: Sep 2, 2015 (Epub ahead of print)

14. Zhang C, Hu Y, Wan J and He H: MicroRNA-124 suppresses the migration and invasion of osteosarcoma cells via targeting ROR2-mediated non-canonical Wnt signaling. Oncol Rep 34: 2195-2201, 2015

15. Xu R, Liu S, Chen $\mathrm{H}$ and Lao L: MicroRNA-30a downregulation contributes to chemoresistance of osteosarcoma cells through activating Beclin-1-mediated autophagy. Oncol Rep 35: 1757-1763, 2016.

16. Wang W, Zhou X and Wei M: MicroRNA-144 suppresses osteosarcoma growth and metastasis by targeting ROCK1 and ROCK2. Oncotarget 6: 10297-10308, 2015.

17. Lin Z, Song D, Wei H, Yang X, Liu T, Yan W and Xiao J: TGF- $\beta 1$-induced miR-202 mediates drug resistance by inhibiting apoptosis in human osteosarcoma. J Cancer Res Clin Oncol 142 239-246, 2016

18. Wang Y, Jia LS, Yuan W, Wu Z, Wang HB, Xu T, Sun JC, Cheng KF and Shi JG: Low miR-34a and miR-192 are associated with unfavorable prognosis in patients suffering from osteosarcoma. Am J Transl Res 7: 111-119, 2015.

19. Yun EJ, Song KS, Shin S, Kim S, Heo JY, Kweon GR, Wu T, Park JI and Lim K: Docosahexaenoic acid suppresses breast cancer cell metastasis by targeting matrix-metalloproteinases. Oncotarget 7: 49961-49971, 2016.

20. Tian X, Ye C, Yang Y, Guan X, Dong B, Zhao M and Hao C: Expression of CD147 and matrix metalloproteinase-11 in colorectal cancer and their relationship to clinicopathological features. J Transl Med 13: 337, 2015.

21. Al-Alem L and Curry TE Jr: Ovarian cancer: Involvement of the matrix metalloproteinases. Reproduction 150: R55-R64, 2015.

22. Basset P, Bellocq JP, Lefebvre O, Noël A, Chenard MP, Wolf C, Anglard P and Rio MC: Stromelysin-3: A paradigm for stroma-derived factors implicated in carcinoma progression. Crit Rev Oncol Hematol 26: 43-53, 1997.

23. Livak KJ and Schmittgen TD: Analysis of relative gene expression data using real-time quantitative PCR and the 2(-Delta Delta C(T)) method. Methods 25: 402-408, 2001
24. Unni KK and Dahlin DC: Grading of bone tumors. Semin Diagn Pathol 1: 165-172, 1984

25. Varshney J and Subramanian S: MicroRNAs as potential target in human bone and soft tissue sarcoma therapeutics. Front Mol Biosci 2: 31, 2015.

26. Fire A, Xu S, Montgomery MK, Kostas SA, Driver SE and Mello CC: Potent and specific genetic interference by double-stranded RNA in Caenorhabditis elegans. Nature 391: 806-811, 1998

27. Bartel DP: MicroRNAs: Genomics, biogenesis, mechanism, and function. Cell 116: 281-297, 2004.

28. Calin GA, Sevignani C, Dumitru CD, Hyslop T, Noch E, Yendamuri S, Shimizu M, Rattan S, Bullrich F, Negrini M and Croce CM: Human microRNA genes are frequently located at fragile sites and genomic regions involved in cancers. Proc Natl Acad Sci USA 101: 2999-3004, 2004.

29. Liang HQ, Wang RJ, Diao CF, Li JW, Su JL and Zhang S: The PTTG1-targeting miRNAs miR-329, miR-300, miR-381, and miR-655 inhibit pituitary tumor cell tumorigenesis and are involved in a p53/PTTG1 regulation feedback loop. Oncotarget 6: 29413-29427, 2015.

30. Esquela-Kerscher A and Slack FJ: Oncomirs-microRNAs with a role in cancer. Nat Rev Cancer 6: 259-269, 2006.

31. Jin Y, Lu J, Wen J, Shen Y and Wen X: Regulation of growth of human bladder cancer by miR-192. Tumour Biol 36: 3791-3797, 2015.

32. Geng L, Chaudhuri A, Talmon G, Wisecarver JL, Are C, Brattain $\mathrm{M}$ and Wang J: MicroRNA-192 suppresses liver metastasis of colon cancer. Oncogene 33: 5332-5340, 2014.

33. Hu F, Meng X, Tong Q, Liang L, Xiang R, Zhu T and Yang S: BMP-6 inhibits cell proliferation by targeting microRNA-192 in breast cancer. Biochim Biophys Acta 1832: 2379-2390, 2013

34. Xiang M, Zeng Y, Yang R Xu H, Chen Z, Zhong J, Xie H, Xu Y and Zeng X: U6 is not a suitable endogenous control for the quantification of circulating microRNAs. Biochem Biophys Res Commun 454: 210-214, 2014.

35. Li S, Li F, Niu R, Zhang H, Cui A, An W and Wang X: Mir-192 suppresses apoptosis and promotes proliferation in esophageal aquamous cell caicinoma by targeting Bim. Int J Clin Exp Pathol 8: 8048-8056, 2015

36. Zhao C, Zhang J, Zhang S, Yu D, Chen Y, Liu Q, Shi M, Ni C and Zhu M: Diagnostic and biological significance of microRNA-192 in pancreatic ductal adenocarcinoma. Oncol Rep 30: 276-284, 2013.

37. Okada A, Saez S, Misumi Y and Basset P: Rat stromelysin 3: cDNA cloning from healing skin wound, activation by furin and expression in rat tissues. Gene 185: 187-193, 1997.

38. Brennecke J, Stark A, Russell RB and Cohen SM: Principles of microRNA-target recognition. PLoS Biol 3: e85, 2015. 